\title{
Long Term Surgical Oncological and Functional Outcome of Large Petroclival and Cerebellopontine Angle Epidermoid Cysts: A Multicenter Study.
}

Aurore Sellier ( $\square$ aurore.sellier@live.fr)

HIA Sainte Anne https://orcid.org/0000-0003-2655-8515

Lucas Troude

Aix Marseille University

Clément Baumgarten

Grenoble University Hospital

Yohan Caudron

Pitié Salpêtrière Hospital

Maxime Bretonnier

Rennes University Hospital

Clémentine Gallet

Angers University Hospital

Sébastien Boissonneau

Aix Marseille University

Pierre-Julien Cungi

HIA Sainte Anne

Xavier Morandi

Rennes University Hospital

Henry Dufour

Aix Marseille University

Henri-Dominique Fournier

Angers University Hospital

Emmanuel Gay

Grenoble University Hospital

Michel Kalamarides

Pitié Salpêtrière Hospital

Pierre-Hugues Roche

Aix Marseille University 
Keywords: Epidermoid Cyst, Petroclival Area, Cerebellopontine Angle, Cranial Nerve, Functional Outcome, Microsurgery

Posted Date: November 1st, 2021

DOI: https://doi.org/10.21203/rs.3.rs-185088/v1

License: (c) (i) This work is licensed under a Creative Commons Attribution 4.0 International License. Read Full License

Version of Record: A version of this preprint was published at Neurosurgical Review on January 10th, 2022. See the published version at https://doi.org/10.1007/s10143-021-01702-2. 


\section{Abstract}

Objective: To assess the long-term surgical results on cranial nerve (CN) function and tumor control in patients harboring cerebellopontine angle (CPA) and petroclival area (PCA) epidermoid cysts (EC).

Methods: This is a retrospective cohort study about 56 consecutive patients operated on for a CPA or PCA EC between January 2001 and July 2019 in six participating French cranial base referral centers.

Results: Sixteen patients (29\%) presented a PCA EC, and 40 a CPA EC (71\%). The median clinical and radiological follow-up was 46 months (range 0-409). Preoperative $\mathrm{CN}$ disorders were present in $84 \%$ of patients ( $n=47), 72 \%$ of them experienced $C N$ deficits improvement at last follow-up consultation ( $n=34)$ : $60 \%$ of cochlear and vestibular deficits ( $n=9 / 15$ in both groups), $67 \%$ of trigeminal neuralgia $(n=10 / 15), 53 \%$ of trigeminal hypoesthesia $(n=8 / 15), 44 \%$ of lower cranial nerve disorders $(n=4 / 9), 38 \%$ of facial nerve deficits $(n=5 / 8)$, and $43 \%$ of oculomotor deficits $(n=3 / 7)$ improved or were cured after surgery. New postoperative $\mathrm{CN}$ deficits occurred in $48 \%$ of patients $(n=27)$. Most of them resolved at last follow-up, except for cochlear deficits which improved in only $14 \%$ of cases $(n=1 / 7)$. Twenty-six patients $(46 \%)$ showed evidence of tumor progression after a median duration of 63 months (range 7-210). Extent of resection, tumor location and tumor size were not associated with the occurrence of new postoperative $\mathrm{CN}$ deficit nor tumor progression.

Conclusion: A functional nerve-sparing resection of posterior fossa EC is an effective strategy to optimize the results on preexisting $\mathrm{CN}$ deficits and reduces the risk of permanent de novo deficits.

\section{Introduction}

Epidermoid cysts (EC), also known as pearly tumors or cholesteatomas, are rare benign tumors that represent 0.2 to $1.8 \%$ of all intracranial tumors.[5] They are congenital lesions caused by abnormal trapping of epidermal elements during the processes of neural groove closure and disjunction of surface ectoderm, which occur between the 3rd and 5th weeks of fetal life.[6] They display a thin epithelial-lined capsule, and their growth is mainly due to the accumulation of desquamated epithelial cells, forming shiny pearly debris, in addition to the secretion of cholesterol and keratin into the cyst. Cerebellopontine angle (CPA) cysts, being the most common location for posterior fossa EC, constitute approximately 40$50 \%$ of all intracranial EC. They represent the third most common CPA tumors after acoustic neuromas and meningiomas, comprising approximately $7 \%$ of CPA pathology.[7, 17]

EC grow slowly and may penetrate to every accessible space around its origin. EC originating from the petroclival area (PCA) and the CPA share common behavior and surgical challenges. Cranial nerve (CN) disorders are the foremost symptoms in both locations. Microsurgical resection is the only effective treatment for these lesions when eligible for an active treatment [15]. Previous reports have already studied the correlation between total removal and tumor recurrence. Some authors found a higher rate of recurrence after non-total resection, $[1,7,19,22]$ while other reports found no difference between GTR and PR. $[13,20]$ Moreover, little is known about the kinetic of regrowth after incomplete surgical resection. 
Some other studies have focused on functional outcomes and the course of $\mathrm{CN}$ deficits after surgery.[3, 4, $9,13,14]$ However, there is no existing report presenting a detailed monitoring of the evolution of $\mathrm{CN}$ deficits over time.

The aim of this multicenter study was to assess the long-term oncological and functional surgical outcome in CPA/PCA EC patients.

\section{Methods}

All consecutive patients who underwent surgical treatment for a CPA or PCA EC between January 2001 and July 2019 in six participating French cranial base referral centers were included in this observational retrospective cohort study. The centers involved were North University Hospital, Grenoble University Hospital, Pitié Salpêtrière Hospital, Rennes University Hospital, Angers University Hospital, La Timone Hospital.

This study has been approved by the French Neurosurgical College Institutional Review Board (reference: IRB00011687 College de neurochirurgie IRB \#1: 2020/04).

\section{Data collection}

Demographics, clinical manifestations (presenting signs and symptoms, duration of symptoms, CN function), radiological investigations (location, extension, laterality, size, existence of a diffusion-weighted magnetic resonance imaging (DWI) sequence, complications), surgical procedure (surgical approach, extent of removal), postoperative imagery, postoperative complications (occurring within 30 days after surgery), neurological outcome, radiological progression/recurrence and possible second surgery were retrospectively reviewed. Tumor size was assessed based on their largest diameter in MRI.

PCA cysts were distinguished from CPA cysts by their primary origin in the upper two-thirds of the clivus or the petrous apex, medial to the trigeminal nerve. Both could then extend forward or backward to the cavernous sinus, the Meckel's cave, the sellar region, the incisure of the tentorium, the porus and the ventral edge of the foramen magnum.

\section{Surgical protocol: Functional-sparing technique}

The main goal of the surgery was to achieve optimal and safe cyst resection, without compromising neurovascular structures (functional sparing technique). The intraoperative decision to interrupt the resection was based on the evidence of critical adhesion of the tumor capsule to $\mathrm{CN}$, vascular structures or brain stem. In this situation, small fragments of capsule were left in place to avoid the risk of neurological deficits. The surgical approach was customized for location and cyst extension and depended on the experience of each center. Neuronavigation and $\mathrm{CN}$ neurophysiological monitoring could be used by the surgeon according to his preoperative evaluation. The suboccipital retrosigmoid (RS)

(Figure 1) and the epidural anterior transpetrosal (TP) approach (Figure 2) were the most frequently used. In case of cyst extension above the tentorium, the resection could be combined with a pterional or a 
subtemporal approach. A median suboccipital telovelar approach could also be performed when the lesion had spread into the fourth ventricle.

\section{Postoperative Assessment}

Clinico-radiological follow-up was planned at 3, 6 and 12 months after resection, and subsequently at 2, $3,5,7 \& 10$ years after surgery, and then once every 3 years thereafter.

The function of each $\mathrm{CN}$ involved in the posterior fossa was analyzed in the postoperative period, and at every follow up visit. The Barrow Neurological Institute (BNI) classification [18] was used to assess the trigeminal neuralgia, and the facial nerve function was scored according to the House and Brackmann (HB) classification.[11] Preoperative $\mathrm{CN}$ deficit improvement was defined as the reduction or resolution of one or more preoperative $\mathrm{CN}$ deficit(s) between the preoperative period and the end of follow up.

The patient's functional status evolution was assessed with the World Health Organization performance status score (WHO PS) [16] at each follow up consultation.

The postoperative cystic residue was assessed by MRI scan at 3 months after surgery. Extent of resection (EOR) was considered total (Gross Total Resection, GTR) if keratinous debris and the entire tumor capsule were removed, confirmed with the absence of postoperative cystic residue in DWI on the first postoperative MRI. Subtotal Resection (STR) was defined as complete content removal and incomplete capsule removal, with a small residue on postoperative MRI. Partial Resection (PR) was defined as incomplete resection of both cyst contents and capsule, with postoperative residue on postoperative MRI.

Progression or recurrence of the lesion was based on the apparition or increase of the EC size on the DWI sequences of the follow-up MRI scan.

$\underline{\text { Statistical analysis }}$

All statistical analyses were conducted using IBM Corp. Released 2012. IBM SPSS Statistics for Windows, Version 25.0. Armonk, NY: IBM Corp.

Categorical variables were presented as numbers and percentages, and continuous variables were presented as mean and standard deviation or median and range (minimum-maximum), as appropriate. Analyses were tailored to address associations among surgical data (surgical approach, EOR, tumor location and tumor size) and the occurrence of preoperative $\mathrm{CN}$ deficit improvement, new postoperative $\mathrm{CN}$ deficit and tumor progression/recurrence. Univariate analyses were performed using the Pearson's chisquared test or Fisher's exact test for categorical variables, and Mann Whitney $U$ test for continuous variables, as appropriate. The progression free survival after surgery and the time between progression and the second surgery were presented as Kaplan-Meier plots. A two-sided p-value of less than 0.05 was considered to indicate statistical significance. Adjusted $p$-value $<0,004$ was considered statistically significant after Bonferroni correction for multiple comparison tests. 


\section{Results}

\section{Population study_}

A total of 56 consecutive patients were enrolled in this study, including 29 (52\%) females and 27 (48\%) males, with a median age of 38 years $(20-71)$ at the time of diagnosis. Six patients $(11 \%)$ had previously been operated on in other centers. The median duration of symptoms before diagnosis was 6 months ( 0 300).

\section{Clinical and radiological features (Table 1).}

Fifty-four patients (96\%) were symptomatic at the time of the diagnosis. Preoperative $\mathrm{CN}$ disorders were present in 47 patients (84\%). The foremost symptoms were the cochleo-vestibular impairment (25 patients - 45\%): 15 patients reported hearing loss, and 15 patients presented with vestibular deficits (balance disorder/tinnitus/vertigo). Fifteen patients (27\%) suffered from trigeminal neuralgia, and 15 patients $(27 \%)$ presented with facial hypoesthesia. Lower cranial nerve (LCN) disorders were observed in 9 patients (16\%). Facial nerve deficits were present in 8 patients (14\%) preoperatively, mostly HB grade II $(11 \%, n=6)$. Seven patients $(13 \%)$ presented one or more oculomotor dysfunction in the preoperative period: there were $3 \mathrm{CN}$ III (5\%), 3 CN IV (5\%) and 5 CN VI (9\%) deficiencies.

Sixteen EC (29\%) were mainly localized in the PCA, and 40 EC (71\%) were localized in the CPA.

\section{Surgical Features (Table 2).}

RS approach was the most frequently performed, especially for the CPA location: 75\% of CPA EC were removed through this approach, $3 \%$ were resected through a pterional approach, $10 \%$ through a TP approach, $10 \%$ through a subtemporal approach and $3 \%$ through a suboccipital approach $(n=30,1,4,4$, $1 / 40$, respectively). In the PCA location, the surgical approach was less homogeneous. A RS approach was performed in $38 \%$ of them, a pterional approach in $44 \%$ of them, a TP approach in $13 \%$ of them, and a subtemporal approach in $7 \%$ of them $(n=6,7,2$ and $1 / 16$, respectively).

GTR, STR and PR were performed in respectively $14 \%(n=8), 61 \%(n=34)$ and $23 \%(n=13)$ of patients. The EOR was assessed by the surgeon at the end of the procedure in all cases, according to the criteria defined above. The surgical perioperative evaluation was also confirmed by a DWI sequence on the 3-month postoperative MRI when available (42 patients -75\%). For the remaining 14 patients, the surgery occurred before 2010 and no 3-month postoperative MRI was performed then.

The most frequent postoperative complications were aseptic meningitis $(13 \%, n=7)$, and hydrocephalus $(7 \%, n=4)$. A 28 -year-old woman died in the postoperative period from a postoperative cerebellar hematoma.

\section{Functional outcome}

\section{Preoperative $\mathrm{CN}$ deficit}


- Course of preoperative $\mathrm{CN}$ deficit (Table 3)

Among the 47 patients with $\mathrm{CN}$ disorders before surgery, 34 experienced a $\mathrm{CN}$ improvement during follow up $(72 \%$, Figure $3 A)$.

At the end of follow-up, $60 \%$ of the cochlear and vestibular preoperative dysfunction improved or were completely resolved ( $n=9 / 15$ in both groups). Six patients retained hearing loss or deafness, and 6 had a persistent vestibular deficit at the end of the follow-up.

Respectively 67 and $53 \%$ of trigeminal preoperative neuralgia and hypoesthesia were resolved at the last follow up control $(n=10 / 15$ and $n=8 / 15)$. Among the remaining five persistent neuralgia ( $9 \%)$, three (5\%) decreased according to the BNI classification (Figure 4).

Preoperative LCN disorders disappeared throughout the follow-up in $44 \%$ of cases $(n=4 / 9)$.

In the same way, an improvement of preexisting facial nerve deficits was seen in $38 \%$ of cases $(n=5 / 8)$.

Forty-three percent of patients with preoperative oculomotor nerve deficit were asymptomatic at the end of follow up ( $n=3 / 7)$, while $57 \%$ of patients $(n=4 / 7)$ retained an oculomotor deficit (CN VI: $n=2, C N$ IV: $\mathrm{n}=1$ and $\mathrm{CN}$ III: $\mathrm{n}=1)$.

- Prediction of preoperative $\mathrm{CN}$ deficit improvement at last follow-up consultation

Eighty-eight percent $(n=7 / 8)$ of patients who received GTR experienced an improvement in their initial CN deficit, compared to $53 \%(n=18 / 34)$ for STR and $69 \%(n=9 / 13)$ for PR $(p=0.196)$. Patients who experienced postoperative improvement of their $\mathrm{CN}$ deficit harbored smaller tumor volumes than those who remained stable over time (median $40 \mathrm{~mm}$ (range 17-72) vs median $45 \mathrm{~mm}$ (range 24-82); $p=0,127$ ). There was no significant association between the surgical approach or the EC location and the improvement of preoperative deficit (respectively $67 \%$ of improvement for TP vs $64 \%$ for RS approach ( $p$ $=1.00)$; and $65 \%$ of improvement for CPA vs $50 \%$ for PCA location $(p=0.369)$ ).

2. New postoperative deficit

\section{- Course of new postoperative CN deficit (Table 4)}

New neurological $\mathrm{CN}$ deficits occurred during the immediate postoperative course in 27 (48\%) patients (Figure 3B). Among the patients with oculomotor CN, vestibular CN and LCN postoperative disorders, only 2 still experienced an oculomotor deficit at the end of follow-up, and none retained LCN or vestibular CN impairment anymore.

In contrast, only $14 \%$ of patients with postoperative cochlear deficit improved at the end of follow-up ( $n=$ 1/7). To resume, 44 patients were free of cochlear deficits at the last follow up examination, including 9 preoperative cochlear deficit and the new postoperative deficit that improved. 
In the univariate analysis, patients who underwent a TP approach had an increased risk of new postoperative deficit compared to the ones treated with a RS approach $(100 \%$ vs $42 \%, p=0.020)$. All of them were oculomotor deficit, including $2 \mathrm{CN}$ III deficits (1 transitory deficit and 1 persistent at discharge), 5 CN IV deficits (4 transitory deficits, and only one persistent at discharge), and $2 \mathrm{CN}$ VI transitory deficits. When a RS approach was performed, the new postoperative oculomotor rate was only $22 \%$ versus $100 \%$ after a TP approach $(p=0.001)$. After Bonferroni correction, significant differences (adjusted $p$ value $<0.004$ ) only existed between TP approach and oculomotor rate. Tumor size, location and extent of resection were not statistically associated with de novo early postoperative $\mathrm{CN}$ deficits (Table 5).

\section{Functional status}

At last follow-up examination, 49 patients (88\%) were fully independent (WHO PS 0 and 1) compared to 45 patients $(80 \%)$ in the postoperative period. The number of ambulatory patients unable to work (WHO PS at 2) decreased over time, with 3 patients (5\%) at the end of follow-up compared to 8 patients (14\%) in the early postoperative period. Only 2 patients displayed poor general condition (WHO PS 3 and 4) at the end of follow-up (4\%). Finally, two patients died during follow-up: one in the postoperative period as described previously, and the other after 45 days of intensive care for postoperative cerebral ischemia. To note, the second comatose patient survived and was ambulatory at the end of follow up (WHO PS at 2).

\section{$\underline{\text { Tumor control }}$}

No patient was lost to follow-up in the first one-year postoperative period, except for deceased patient._The median clinical and radiological follow-up was 46 months (0-409). Twenty-six patients (46 $\%$ ) showed evidence of tumor progression or recurrence during the follow-up period, after a median duration of 63 months $(7-210)$. Fifty percent $(n=13)$ of the patients presenting with EC progression underwent a second surgery. Re-operated patients were symptomatic in $85 \%$ of cases before second surgery $(n=11 / 13)$. The remaining two patients were operated on again because of the rapidity of the cyst expansion. The median delay between the recurrence and the new surgery was 5 months (0-41) (Figure 5).

RS approach and smaller tumor size tended to be associated with less recurrences $(p=0.672$ and 0.245 respectively, see Table 5). Postoperative residual volume was not associated with tumor progression or recurrence: $50 \%$ of GTR patients experienced tumor regrowth, as well as $50 \%$ of STR patients and $31 \%$ of PR patients $(p=0.394)$. Similarly, CPA or PCA location of the EC did not influence the recurrence rate $(p=$ 1).

\section{Discussion}

The kinetics of postoperative recovery of $\mathrm{CN}$ disorders is an original aspect of this work, which demonstrates the favorable effect of surgery on the functional outcome of $\mathrm{CN}$ function, with a low rate of 
long-term morbidity since postoperative $\mathrm{CN}$ deficits were mostly transient.

This study is one of the largest surgical series of CPA-PCA EC published so far, with a total of 56 patients. The literature dealing with the same topic is scarce and only a few retrospective cohort studies include more than 30 patients. $[5,7,12,13,19,23,24]$

\section{CN preoperative deficit}

We report here the very good evolution of preoperative $\mathrm{CN}$ disorders after surgery, with an improvement of $72 \%$ of preexisting deficits, regardless of the approach or the location (PCA or CPA) of the cyst.

Both the inflammatory effect induced by the cystic content and the direct mechanical compression over the cisternal segment of the $\mathrm{CN}$ by the tumor, may explain preoperative $\mathrm{CN}$ disorders favorable outcome after surgery, even with small volume cysts. Contrary to schwannoma or meningioma cases wherein the $\mathrm{CN}$ are shift by the boundaries of the tumor capsule, EC invade the cisternal space by adapting their shape to the local morphology, encompassing nerves and vessels. Thus, surgery might improve symptoms by reducing cystic content and inflammatory process over the nerve in addition to reducing the mass effect. In this way, GTR has a greater impact than STR and PR on the improvement of pre-operative CN deficits in our series ( $88 \%$ of improvement VS $53 \%$ and $69 \%, p=0.196)$ : maximal resection of cystic content and capsule fragments results in a higher reduction of local irritation. The effects of local irritation by the cholesterol seeping through the cyst wall have already been reported in previous study, in cases of hyperactive dysfunction such as trigeminal neuralgia or hemifacial spasm. [5, 9] Vascular compression of the nerve, either by a displaced artery or by nerve displacement toward the artery by the tumor has also been relieved. $[5,13]$

The beneficial effects of surgery on these preoperative $\mathrm{CN}$ deficits had also been demonstrated in several series. Of the 17 CPA EC reported by Czernicky et al, 11 patients experienced improvement or resolution of their preoperative deficits, in particular with trigeminal neuralgia, LCN deficits and facial nerve deficits.[4] In their cohort of 37 EC patients, Gopalakrishnan et al demonstrated a significant improvement in trigeminal and lower cranial nerve dysfunction after surgery, and half of the CN VIII and oculomotor deficits [7]. Of the 21 patients with preoperative $\mathrm{CN}$ dysfunction reviewed by Schiefer and Link, 33\% were resolved $(n=7)$ after the surgery and $43 \%$ were improved $(n=9)$. [20] In Vernon et al series of 139 patients, $74 \%$ of them improved compared with their preoperative clinical status. Prior to our series, none of these studies attempted to analyze the association between these $\mathrm{CN}$ improvements and surgical approach or EOR. Of note, none of them included PCA location of the cyst, and the TP approach was not used.

\section{Postoperative new CN deficit}

New $\mathrm{CN}$ deficits occurred during the immediate postoperative course in almost $50 \%$ of our patients. Most of them, apart from cochlear impairment, tended to resolve during the follow-up period. The unique cisternal cytoarchitecture of the VIII CN (i.e. centrally myelinated) could explain its higher surgical 
vulnerability in comparison to the other $\mathrm{CN}$. Moreover, postoperative $\mathrm{CN}$ VIII impairment is related to direct nerve dissection or vasculature damage during surgery because of the adhesion between the lesion and the nerve and is therefore less likely to recover.[8]

TP approach tended to increase postoperative impairment, especially oculomotor deficits $(p=$ 0.001). However, most of them were transient and TP approach remains advantageous for some PCA locations, particularly when the cyst crosses the midline or straddles the basilar artery. Thus, this approach must remain systematically considered to ensure the best surgical exposure.

The high rate of postoperative $\mathrm{CN}$ injury, resulting from the adhesion of the tumor capsule to the nerve, is a well-known complication of this surgery.[12] Very few studies have detailed the evolution of CN deficit over time. $[4,5,7,12,13,20,23]$. Vernon et al reported $41 \%$ of new postoperative deficits, which resolved completely on long-term follow-up in $21 \%$ patients, improved significantly in $10 \%$ patients, and remained at an unchanged level in $9 \%$ patients. Czernicki et al provided comparable results in term of frequency and evolution of $\mathrm{CN}$ deficit, with $58 \%$ of new postoperative $\mathrm{CN}$ injury $(\mathrm{n}=10)$ and persistent cochlear deficits during follow-up. Similar to our series, the EOR had no effect on the occurrence of new postoperative deficits.[4] In Gopalakrishnan series of CPA EC, only $13 \%(n=5)$ of the 38 new postoperative deficits persisted at long term follow-up.[7] They reported a higher incidence of new neurological deficits in patients undergoing total removal compared to subtotal removal, but at the same time, the former group experienced a better improvement in preoperative neurological deficits compared to the latter one.[7] Two series have reported a lower rate of postoperative deficit.[5, 12] The cohort of 30 patients undergoing retrosigmoid surgery associated with whole course neuroendoscopy of Hu et al experienced only $7 \%$ of new deficits.[12] Finally, two papers have reported a higher rate of new postoperative deficit, but with a very good improvement over time.[13, 20] Despite these differences in term of postoperative $\mathrm{CN}$ deficit, our cohort covered a larger group of accurately and sequentially monitored patients than previously reported, which was the main objective of this work, and strengthen our findings.

\section{General Condition}

Long term general condition was good to excellent for most of patients. Only 2 patients presented a WHO PS score at 3 or 4 (capable of limited self-care or completely disabled) at the end of follow-up. In the literature, two other series have recorded long-term general condition, and their results were similar.[4, 20] Eighty-eight percent of patients were able to carry out all usual activities (modified Rankin score of 0 or 1 ) in Czernicki's cohort, and $84 \%$ for Schiefer's cohort. These results are linked to a cautious surgical strategy, avoiding maximalist resection of the fragments adhering to the brain stem, vessels or nerves. The fact that EC are mostly managed in young and healthy patients favorably outweigh the outcome.

\section{Tumor control and onco-functional balance}

Twenty-six patients (46\%) showed evidence of tumor progression during the follow-up period, after a median duration of 63 months. The factors that might predict a stable behavior instead of a keep 
growing ones couldn't be unveiled by our study. Based on our experience, patients with postoperative residual lesions had no increased risk of progression or recurrence during follow-up $(p=0.394)$. This result could be due to many biases inherent to the design of the study, and to the short follow-up of only 46 months. In our work, GTR rate (14\%) is lower and recurrence rate (46\%) higher than those previously reported for posterior fossa EC: Farhoud et al (32 patients, 59\% GTR rate, 0 recurrence), Samii et al (40 patients, $75 \%$ GTR rate, $8 \%$ recurrence rate), Kobata et al (30 patients, 57\% GTR rate, $7 \%$ recurrence rate), Vernon et al (139 patients, 73\% GTR rate, $8 \%$ recurrence rate), Yawn et al (47 patients, 46\% GTR rate, $8 \%$ recurrence rate) or Schiefer et al (24 patients, $54 \%$ GTR rate, $25 \%$ recurrence rate) for instance. $[5,13,19$, $20,23,24]$ Recurrence rate in Gopalakrishnan et al series was higher after long term follow up : $45 \%$ of patients showed evidence of tumor recurrence after a mean duration of 9.3 year.[7] Indeed, our definitions of GTR and recurrence were strict, and non-total resections and recurrence rate could have been overestimated.

Postoperative tumor control results are heterogeneous in the literature. Like our study, some reports found no difference in tumor progression after complete or incomplete resection, even after longer follow-up periods (respectively 11,5 years and 4,3 years of follow up). $[13,20]$ In contrast, some authors found a higher rate of recurrence after non-total resection [7, 19, 22]. A recent large meta-analysis including 691 patients with intracranial epidermoid tumors found that STR was associated with a 7-times higher rate of regrowth than tumors that underwent GTR.[22] However, the analyses were not stratified according to the intracranial location of the cysts, such as infratentorial sites. Moreover, capsule adherence to the neurovascular structures in PCA and CPA locations makes total removal extremely challenging. Additionally, the propensity of EC for regional spreading toward neighbor cisterns hampers the ability to expose the full volume of the cyst "behind the corner" using regular approaches. The use of angled endoscopes (endoscopic assisted microsurgery) could be of help to check for fragments that could be overlooked under microscope, as suggested above.[10,12]

These findings confirm that altogether, operative findings and high field MR doesn't reach the level of sensibility to insure the cure of the disease. EC grow linearly, not exponentially,[2] and the overlooked micro-fragments of cyst walls have the propensity to regrowth which is empirically known. $[4,5,9,12,14$, $20,21]$

Our results (see Kaplan Meier plot, Figure 5A) underline that recurrence or regrowth might be expected in the ultra-late period, regardless the EOR of the cyst. Patients should be aware and a clinic-radiological sequential follow-up must be planned in long term. Only half of the growing residual tumors justified additional surgery (see Kaplan Meier plot, Figure 5B). The expectation for clinical symptomatology in $85 \%$ of reoperated patients can explain the half-year delay before considering surgery.

\section{$\underline{\text { Weaknesses }}$}

This is a retrospective study, and so there may be missing data in our work. Also, the center effect has not been tested. Multivariate analysis and cox-regression analysis was not performed, because not appropriate to this limited patient sample. We deliberately merged the findings of PCA and CPA EC in our 
work; these locations are not supposed to carry the same risks when surgically approached. However, testing this variable did not impact the functional nor the oncological results.

Finally, it would have been interesting to obtain precise volumes and growth rates of the preoperative and residual EC. DWI sequence is essential for the diagnosis of EC and differentiation from other lesions, as the content of the epidermoid cysts shows prominent diffusion restriction due to layered microstructure of the debris [4]. However, this sequence is mediocre in term of anatomical resolution, and did not allow precise calculation of cyst size. The interest of new sequences coupled to the DWI in order to increase the three-dimensional resolution while keeping an important sensitivity and specificity for the diagnosis of EC is an axis to develop in the future.

Last, the length of follow-up did not reach the long-term which weaken the analysis of tumor growth potential over time.

\section{Conclusion}

This multicenter retrospective cohort study of large posterior fossa EC demonstrates the favorable impact of a functional sparing surgery policy over preexisting $\mathrm{CN}$ deficits. In addition, the high rate of early new postoperative $\mathrm{CN}$ deficits observed in this study underlines the complexity of such surgery. Most of these disturbances recovered, except for the cochlear nerve. The counterpart of this surgical strategy was the evidence of tumor remnant or recurrence in half of the study group, which was demonstrated by a regular sequential MR follow-up protocol. The linear progression of EC and the need of additional surgery in half of recurrent patients supports the need for a lifetime DWI MR surveillance, which need to be early mentioned to the patient.

\section{Abbreviations}

$\mathrm{BNI}=$ Barrow Neurological Institute

$\mathrm{CPA}=$ Cerebellopontine angle

$\mathrm{CN}=$ Cranial nerve(s)

DWI = Diffusion-weighted magnetic resonance imaging

$\mathrm{EC}=$ Epidermoid cyst

EOR $=$ Extent of resection

GTR $=$ Gross total resection

$\mathrm{HB}=$ House and Brackmann

LCN $=$ Lower cranial nerves 
$\mathrm{PCA}=$ Petroclival area

PFS = Progression-free survival

$\mathrm{PR}=$ Partial resection

RS = Suboccipital retrosigmoid

STR $=$ Subtotal Resection

TP = Transpetrosal

WHO PS = World Health Organization performance status

\section{Declarations}

On behalf of all authors, the corresponding author states that there is no conflict of interest.

Funding: None

Conflicts of interest/Competing interests: The authors report no conflict of interest.

Availability of data and material: Data will be available upon reasonable request from a qualified investigator.

Code availability: Not applicable

Ethics approval: This study has been approved by the French Neurosurgical College Institutional Review Board (reference: IRB00011687 College de neurochirurgie IRB \#1: 2020/04).

Consent to participate: No informed consent was required, as this is a retrospective analysis without any traceable patient data.

Consent for publication: Not applicable.

Authors' contributions:

Author contributions to the study and manuscript preparation include the following: Conception and design: Roche. Acquisition of data: Sellier, Baumgarten, Caudron, Bretonnier, Gallet, Boissonneau. Analysis and interpretation of data: all authors. Drafting the article: Sellier, Troude and Roche. Critically revising the article: all authors. Reviewed submitted version of manuscript: all authors. Approved the final version of the manuscript on behalf of all authors: Roche. Study supervision: Roche.

\section{References}


1. Aboud E, Abolfotoh M, Pravdenkova S, Gokoglu A, Gokden M, Al-Mefty O (2015) Giant intracranial epidermoids: is total removal feasible? J Neurosurg 122:743-756. doi: 10.3171/2014.11.JNS1481

2. Alvord EC (1977) Growth rates of epidermoid tumors. Ann Neurol 2:367-370. doi: 10.1002/ana.410020504

3. Berger MS, Wilson CB (1985) Epidermoid cysts of the posterior fossa. J Neurosurg 62:214-219. doi: 10.3171/jns.1985.62.2.0214

4. Czernicki T, Kunert P, Nowak A, Wojciechowski J, Marchel A (2016) Epidermoid cysts of the cerebellopontine angle: Clinical features and treatment outcomes. Neurol Neurochir Pol 50:75-82. doi: 10.1016/j.pjnns.2015.11.008

5. Farhoud A, Khedr W, Aboul-Enein H (2018) Surgical Resection of Cerebellopontine Epidermoid Cysts: Limitations and Outcome. J Neurol Surg Part B Skull Base 79:167-172. doi: 10.1055/s-00371606220

6. Forbes JA, Banu M, Lehner K, Ottenhausen M, La Corte E, Alalade AF, Ordóñez-Rubiano EG, Greenfield JP, Anand VK, Schwartz TH (2018) Endoscopic endonasal resection of epidermoid cysts involving the ventral cranial base. J Neurosurg 1-10. doi: 10.3171/2017.12.JNS172575

7. Gopalakrishnan CV, Ansari KA, Nair S, Menon G (2014) Long term outcome in surgically treated posterior fossa epidermoids. Clin Neurol Neurosurg 117:93-99. doi: 10.1016/j.clineuro.2013.11.025

8. Guclu B, Meyronet D, Simon É, Streichenberger N, Sindou M, Mertens P (2009) Anatomie structurelle des nerfs crâniens (V, VII, VIII, IX, X). Neurochirurgie 55:92-98. doi: 10.1016/j.neuchi.2009.02.001

9. Hasegawa M, Nouri M, Nagahisa S, Yoshida K, Adachi K, Inamasu J, Hirose Y, Fujisawa H (2016) Cerebellopontine angle epidermoid cysts: clinical presentations and surgical outcome. Neurosurg Rev 39:259-267. doi: 10.1007/s10143-015-0684-5

10. Hitti FL, Lee JYK (2019) Endoscopic Resection of a Cerebellopontine Angle Epidermoid Cyst via a Retrosigmoid Approach. J Neurol Surg Part B Skull Base 80:S330. doi: 10.1055/s-0039-1677852

11. House JW, Brackmann DE (1985) Facial nerve grading system. Otolaryngol--Head Neck Surg Off J Am Acad Otolaryngol-Head Neck Surg 93:146-147. doi: 10.1177/019459988509300202

12. Hu Z, Guan F, Kang T, Huang H, Dai B, Zhu G, Mao B, Kang Z (2015) Whole Course Neuroendoscopic Resection of Cerebellopontine Angle Epidermoid Cysts. J Neurol Surg Part Cent Eur Neurosurg 77:381-388. doi: 10.1055/s-0035-1558818

13. Kobata $\mathrm{H}$, Kondo A, Iwasaki K (2002) Cerebellopontine angle epidermoids presenting with cranial nerve hyperactive dysfunction: pathogenesis and long-term surgical results in 30 patients. Neurosurgery 50:276-286

14. Miller ME, Mastrodimos B, Cueva RA (2012) Hearing preservation in management of epidermoids of the cerebellopontine angle: CPA epidermoids and hearing preservation. Otol Neurotol 33:1599-1603

15. de Morais MV, Mota RA de AA, Marques TA, Loduca RD de S, Melo PM de P (2019) Epidermoid Cyst in the Cerebellopontine Angle: Technical Description Video. J Neurol Surg Part B Skull Base 80:S325-S326. doi: 10.1055/s-0038-1676997 
16. Oken M, Creech R, Tormey D, Horton J, Davis T, McFadden E, Carbone P (1982) Toxicity and response criteria of the Eastern Cooperative Oncology Group. Am J Clin Oncol 5:649-656

17. Pojskić M, Arnautović KI (2019) Microsurgical Resection of the Epidermoid Tumor in the Cerebellopontine Angle. J Neurol Surg Part B Skull Base 80:S327-S328. doi: 10.1055/s-00381677499

18. Rogers CL, Shetter AG, Fiedler JA, Smith KA, Han PP, Speiser BL (2000) Gamma knife radiosurgery for trigeminal neuralgia: the initial experience of the Barrow Neurological Institute. Int J Radiat Oncol 47:1013-1019. doi: 10.1016/S0360-3016(00)00513-7

19. Samii M, Tatagiba M, Piquer J, Carvalho GA (1996) Surgical treatment of epidermoid cysts of the cerebellopontine angle. J Neurosurg 84:14-19. doi: 10.3171/jns.1996.84.1.0014

20. Schiefer TK, Link MJ (2008) Epidermoids of the cerebellopontine angle: a 20-year experience. Surg Neurol 70:584-590. doi: 10.1016/j.surneu.2007.12.021

21. Schroeder HWS, Oertel J, Gaab MR (2004) Endoscope-assisted microsurgical resection of epidermoid tumors of the cerebellopontine angle. J Neurosurg 101:227-232. doi: 10.3171/jns.2004.101.2.0227

22. Shear BM, Jin L, Zhang Y, David WB, Fomchenko El, Erson-Omay EZ, Huttner A, Fulbright RK, Moliterno J (2020) Extent of resection of epidermoid tumors and risk of recurrence: case report and meta-analysis. J Neurosurg 133:291-301. doi: 10.3171/2019.4.JNS19598

23. Vernon $\mathrm{V}$, Naik $\mathrm{H}$, Guha A Surgical management of cerebellopontine angle epidermoid cysts: an institutional experience of 10 ye. 11

24. Yawn RJ, Patel NS, Driscoll CL, Link MJ, Haynes DS, Wanna GB, Thompson RC, Carlson ML (2016) Primary Epidermoid Tumors of the Cerebellopontine Angle: A Review of 47 Cases. Otol Neurotol Off Publ Am Otol Soc Am Neurotol Soc Eur Acad Otol Neurotol 37:951-955. doi:

10.1097/MAO.0000000000001085

\section{Tables}

Table 1. Clinical data and tumor characteristics 


\begin{tabular}{|c|c|}
\hline Characteristics & Number of patients (\%) - Cl95\% \\
\hline \multicolumn{2}{|l|}{ Initial signs and symptoms } \\
\hline Facial weakness & 7 (12.5\%) Cl95\% (5.4; 21.4$)$ \\
\hline Hemifacial spasm & $4(7.1 \%)$ Cl95\% $(1.8 ; 14.3)$ \\
\hline Facial hypoesthesia & 15 (26.8) Cl95\%(15.8; 40.3) \\
\hline Trigeminal neuralgia & 15 (26.8) Cl95\%(15.8; 40.3) \\
\hline Hearing impairment & 15 (26.8) Cl95\%(15.8; 40.3) \\
\hline Diplopia & 8 (14.3) Cl95\%(5.4; 25) \\
\hline Tinnitus & 9 (16.1) Cl95\%(7.1; 26.8) \\
\hline Vertigo & 15 (26.8) Cl95\%(15.8; 40.3) \\
\hline Gait disturbance & 13 (23.2) Cl95\%(12.5; 33.9) \\
\hline Axial sign & 12 (21.4) Cl95\%(10.7; 32.1) \\
\hline Intracranial hypertension $(\mathrm{ICH})$ & 8 (14.3) Cl95\%(5.4; 23.2) \\
\hline Seizures & 3 (5.4) Cl95\%(0; 12.5) \\
\hline Dysphagia/ Phonation Disorders & 9 (16.1) Cl95\%(7.1; 26.8) \\
\hline \multicolumn{2}{|l|}{ Main location of the lesion } \\
\hline CPA & 16 (28.6) CI95\%(17.9; 41.1) \\
\hline PCA & 40 (71.4) Cl95\%(58.9; 82.1) \\
\hline \multicolumn{2}{|l|}{ Accessory location } \\
\hline Meckel & 11 (19.6) Cl95\%(10.7; 30.4) \\
\hline Foramen Magnum & 2 (3.6) $\mathrm{Cl} 95 \%(0 ; 8.9)$ \\
\hline Supratentorial & 12 (21.4) Cl95\%(10.7; 32.1) \\
\hline Suprasellar & 3 (5.4) Cl95\%(0; 12.5) \\
\hline \multicolumn{2}{|l|}{ Side } \\
\hline Left & 27 (48.2) Cl95\%(35.7; 60.7) \\
\hline Right & 29 (51.8) Cl95\%(39,3; 64.3) \\
\hline Largest diameter $(\mathrm{mm})$ & 44 (IQR $30-50$, range 17-82) \\
\hline Hydrocephalus & 5 (8.9) Cl95\%(1.8; 16.1) \\
\hline Preoperative exploration by DWI sequence & 39 (69.6) Cl95\%(57.1; 80.4) \\
\hline
\end{tabular}


$\mathrm{CPA}=$ Cerebellopontine Angle

$\mathrm{PCA}=$ Petroclival area

Table 2. Surgical and postoperative data

\begin{tabular}{|c|c|}
\hline Characteristics & Number of patients (\%) - Cl95\% \\
\hline \multicolumn{2}{|l|}{ Surgical Approach } \\
\hline RS & 36 (64.3) - Cl95\%(51.8; 76.8) \\
\hline TP & 6 (10.7) - Cl95\%(3.6 ; 19.6) \\
\hline Pterional & 8 (14.3) Cl95\%(5.4; 23.2) \\
\hline Subtemporal & 4 (7.1) Cl95\%(1.8; 14.3) \\
\hline Suboccipital median & $1(1.8)-\mathrm{Cl} 95 \%(0 ; 5.4)$ \\
\hline \multicolumn{2}{|l|}{ Extent of resection } \\
\hline GTR & 8 (14.3) Cl95\%(5.4; 23.2) \\
\hline STR & 34 (60.7) Cl95\%(48.2; 73.2) \\
\hline PR & 13 (23.2) Cl95\%(12.5; 35.7) \\
\hline \multicolumn{2}{|l|}{ Postoperative complications * } \\
\hline Aseptic Meningitis & 7 (12.5) - Cl95\%(5.4; 21.4) \\
\hline Hydrocephalus & 4 (7.1) - Cl95\%(1.8; 14.3) \\
\hline Cerebral Ischemia & $3(5.4)-\mathrm{Cl} 95 \%(0 ; 12.5)$ \\
\hline Infield hematoma & $3(5.4)-\mathrm{Cl} 95 \%(0 ; 12.5)$ \\
\hline Septic meningitis & $3(5.4)-\mathrm{Cl} 95 \%(0 ; 125)$ \\
\hline CSF leakage & $3(5.4)-\mathrm{Cl} 95 \%(0 ; 12.5)$ \\
\hline Coma/ Intensive Care Management & $2(3.6)-\mathrm{Cl} 95 \%(0 ; 8.9)$ \\
\hline Bed rest complication & $2(3.6)-\mathrm{Cl} 95 \%(0 ; 8.9)$ \\
\hline Scar disorder & 2 (3.6) - Cl95\%(0;8.9) \\
\hline Keratitis/ corneal ulceration & $1(1.8)-\quad C \mid 95 \%(0 ; 5.4)$ \\
\hline Death & $1(1.8)-\quad \mathrm{Cl} 95 \%(0 ; 5.4)$ \\
\hline
\end{tabular}

*Complications are considered postoperative when occurring within 30 days after surgery.

RS = Retrosigmoid 
$\mathrm{TP}=$ Transpetrosal

GTR $=$ Gross Total Resection

STR $=$ Subtotal Resection

$\mathrm{PR}=$ Partial Resection

CSF = Cerebro-spinal fluid

Table 3. Preoperative $\mathrm{CN}$ deficits evolution after the surgery

\begin{tabular}{llllll} 
CN Deficit & Preoperatively, & $\begin{array}{l}\text { Immediate } \\
\text { postoperative } \\
\text { care, } \mathbf{n}(\%)\end{array}$ & $\begin{array}{l}6 \text { Months } \\
\text { postoperative, }\end{array}$ & $\begin{array}{l}1 \text { Year } \\
\text { postoperative, }\end{array}$ & $\begin{array}{l}\text { Last } \\
\text { Follow-Up, } \\
\mathbf{n}(\%)\end{array}$ \\
\hline Oculomotor & $7(12.5 \%)$ & $6(10.7 \%)$ & $5(8.9 \%)$ & $4(7.1 \%)$ & $4(7.1 \%)$
\end{tabular}

\section{Trigeminal}

\section{Neuralgia}

\begin{tabular}{llllll} 
BNI2 & $8(14.3 \%)$ & $5(8.9 \%)$ & $4(7.1 \%)$ & $2(3.6 \%)$ & $2(3.6 \%)$ \\
\hline BNI3 & $4(7.1 \%)$ & $5(8.9 \%)$ & $4(7.1 \%)$ & $1(1.8 \%)$ & $1(1.8 \%)$ \\
\hline BNI4 & $3(5.4 \%)$ & 0 & 0 & $2(3.6 \%)$ & $2(3.6 \%)$ \\
\hline Total & $15(26.8 \%)$ & $10(17.9 \%)$ & $8(14.3 \%)$ & $5(8.9 \%)$ & $5(8.9 \%)$ \\
\hline Hypoesthesia & $15(26.8 \%)$ & $15(26.8 \%)$ & $9(16.1 \%)$ & $7(12.5 \%)$ & $7(12.5 \%)$ \\
\hline
\end{tabular}

\section{Facial}

\begin{tabular}{llllll}
\hline H\&B II & $6(10.7 \%)$ & $4(7.1 \%)$ & $2(3.6 \%)$ & $2(3.6 \%)$ & $2(3.6 \%)$ \\
\hline H\&B III & $1(1.8 \%)$ & $1(1.8 \%)$ & $1(1.8 \%)$ & $1(1.8 \%)$ & $1(1.8 \%)$ \\
\hline H\&B IV-VI & $1(1.8 \%)$ & $1(1.8 \%)$ & $1(1.8 \%)$ & 0 & 0 \\
\hline Total & $8(14.2 \%)$ & $6(10.7 \%)$ & $4(7.1 \%)$ & $3(5.4 \%)$ & $3(5.4 \%)$
\end{tabular}

\section{Cochlear}

\begin{tabular}{llllll} 
Hearing loss & $15(26.8 \%)$ & $9(16.1 \%)$ & $6(10.7 \%)$ & $5(8.9 \%)$ & $4(7.1 \%)$ \\
Deafness & 0 & $2(3.6 \%)$ & $2(3.6 \%)$ & $2(3.6 \%)$ & $2(3.6 \%)$ \\
\hline Vestibular & $15(26.8 \%)$ & $9(16.1 \%)$ & $6(10.7 \%)$ & $6(10.7 \%)$ & $6(10.7 \%)$ \\
\hline LCN & $9(16.1 \%)$ & $7(12.5 \%)$ & $5(8.9 \%)$ & $4(7.1 \%)$ & $5(8.9 \%)$
\end{tabular}

BNI =Barrow Neurological Institute Classification 
$\mathrm{H} \& \mathrm{~B}=$ House and Brackmann Scale

LCN = Lower Cranial Nerves

Table 4. New CN postoperative deficits and their evolution

\begin{tabular}{llllll} 
CN Deficit & Preoperatively, & $\begin{array}{l}\text { Immediate } \\
\text { postoperative } \\
\text { care, } \mathbf{n}(\%)\end{array}$ & $\begin{array}{l}\mathbf{6} \text { Months } \\
\text { postoperative, } \\
\mathbf{n}(\%)\end{array}$ & $\begin{array}{l}\mathbf{1} \text { Year } \\
\text { postoperative, } \\
\mathbf{n}(\%)\end{array}$ & $\begin{array}{l}\text { Last } \\
\text { Follow-Up, } \\
\mathbf{n}(\%)\end{array}$ \\
\hline Oculomotor & & $18(32.1 \%)$ & $6(10.7 \%)$ & $2(3.6 \%)$ & $2(3.6 \%)$ \\
\hline CN III & NA & $7(13.2 \%)$ & $1(1.9 \%)$ & $1(1.9 \%)$ & $2(3.8 \%)$ \\
\hline CN IV & NA & $10(18.9 \%)$ & $4(7.5 \%)$ & $1(1.9 \%)$ & $1(1.9 \%)$ \\
\hline CN VI & NA & $6(11.8 \%)$ & $2(3.9 \%)$ & 0 & 0
\end{tabular}

\section{Trigeminal}

\begin{tabular}{llllll}
\hline Neuralgia & NA & 0 & 0 & 0 & 0 \\
\hline Hypoesthesia & NA & $4(7.1 \%)$ & $1(1.8 \%)$ & $1(1.8 \%)$ & $1(18 \%)$ \\
\hline Facial & NA & $2(3.6 \%)$ & $1(1.8 \%)$ & 0 & 0
\end{tabular}

\section{Cochlear}

\begin{tabular}{llllll}
\hline Hearing loss & NA & $4(7.2 \%)$ & $4(7.2 \%)$ & $4(7.2 \%)$ & $3(5.4 \%)$ \\
\hline Deafness & NA & $3(5.4 \%)$ & $3(5.4 \%)$ & $3(5.4 \%)$ & $3(5.4 \%)$ \\
\hline Total & NA & $7(12.5 \%)$ & $7(12.5 \%)$ & $7(12.5 \%)$ & $6(10.7 \%)$ \\
\hline Vestibular & NA & $2(3.6 \%)$ & $1(1.8 \%)$ & 0 & 0 \\
\hline LCN & NA & $6(10.7 \%)$ & $1(1.8 \%)$ & $1(1.8 \%)$ & 0
\end{tabular}

BNI =Barrow Neurological Institute Classification

LCN $=$ Lower Cranial Nerves

$\mathrm{NA}=$ not applicable

LCN $=$ Lower Cranial Nerves

Table 5. Predictive factors

* Univariate analyses: Pearson's chi-squared test, Fisher's exact test or Mann Whitney U test, as appropriate 


\begin{tabular}{|c|c|c|c|c|c|c|}
\hline \multirow[t]{2}{*}{ Predictive factors } & \multicolumn{3}{|c|}{ New postoperative deficit } & \multicolumn{3}{|c|}{ Progression of the lesion } \\
\hline & $\begin{array}{l}\text { Absence } \\
n=29\end{array}$ & $\begin{array}{l}\text { Presence } \\
\mathrm{n}=27\end{array}$ & $\begin{array}{l}P \text { - } \\
\text { value* }\end{array}$ & $\begin{array}{l}\text { No } \\
n=30\end{array}$ & $\begin{array}{l}\text { Yes } \\
n=26\end{array}$ & $\begin{array}{l}P- \\
\text { value* }\end{array}$ \\
\hline \multicolumn{7}{|l|}{ Surgical approach } \\
\hline TP & 0 & 6 & \multirow[t]{2}{*}{0.020} & 3 & 3 & 0.672 \\
\hline RS & 21 & 15 & & 22 & 14 & \\
\hline \multicolumn{7}{|l|}{ Extent of resection } \\
\hline GTR & 5 & 3 & \multirow{3}{*}{0.681} & 4 & 4 & 0.394 \\
\hline STR & 17 & 17 & & 17 & 17 & \\
\hline PR & 7 & 6 & & 9 & 4 & \\
\hline \multicolumn{7}{|l|}{ Location } \\
\hline CPA & 21 & 19 & 1 & 21 & 19 & 1 \\
\hline PCA & 8 & 8 & & 9 & 7 & \\
\hline $\begin{array}{l}\text { Largest diameter } \\
\text { (median, range) }\end{array}$ & $\begin{array}{l}42(19- \\
72)\end{array}$ & $\begin{array}{l}45(17- \\
82)\end{array}$ & 0.478 & $\begin{array}{l}45(19- \\
72)\end{array}$ & $\begin{array}{l}34(17- \\
82)\end{array}$ & 0.245 \\
\hline
\end{tabular}

RS $=$ Retrosigmoid

$\mathrm{TP}=$ Transpetrosal

GTR $=$ Gross Total Resection

STR = Subtotal Resection

PR = Partial Resection

$\mathrm{CPA}=$ Cerebellopontine Angle

PCA $=$ Petroclival area

Figures 

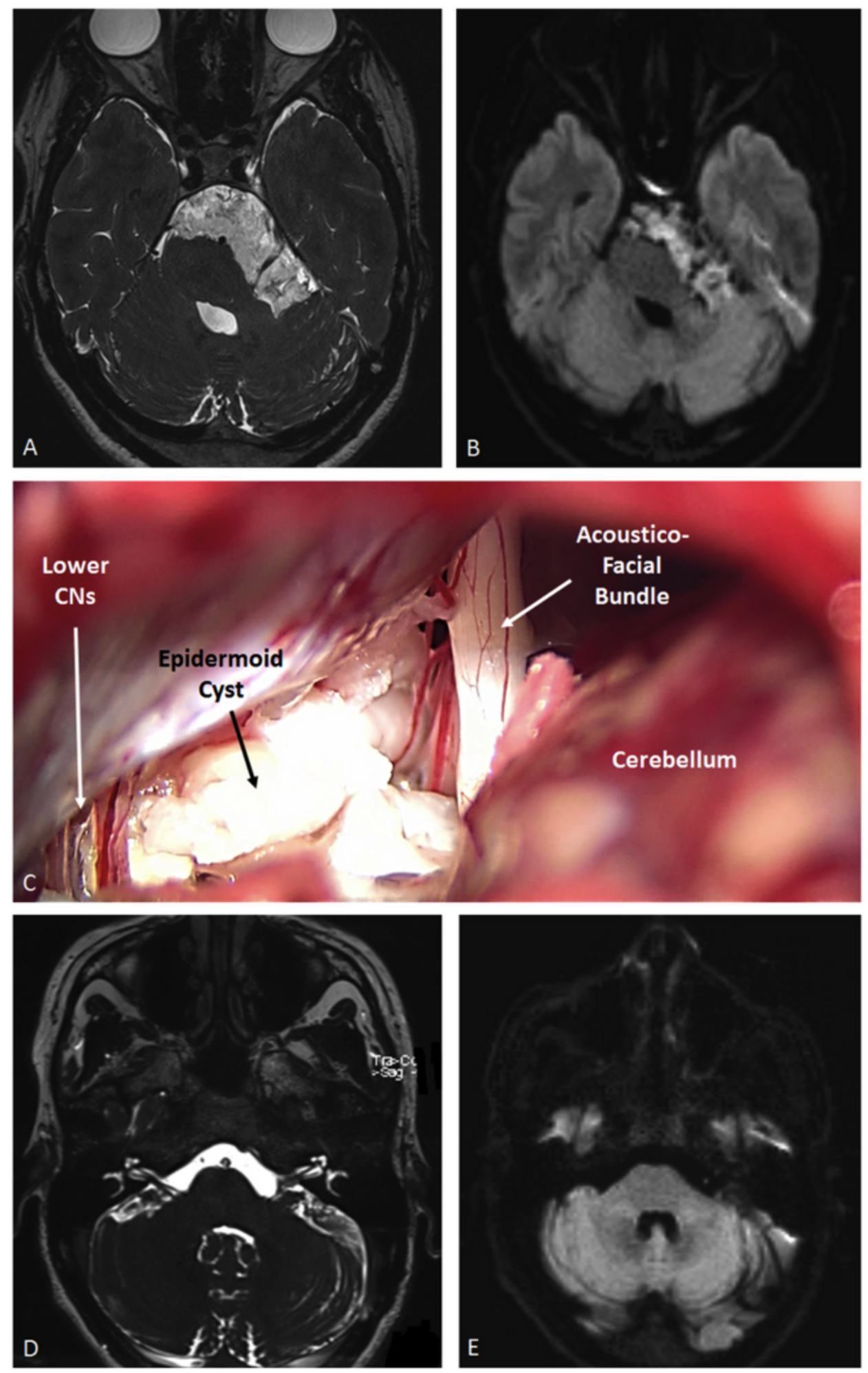

\section{Figure 1}

Illustrative case of a cerebello-pontine angle epidermoid cyst operated on through a retrosigmoid approach A \& B: The preoperative DWI Diffusion and T2-weighted MRI showed a left cerebellopontinelllustrative case of a cerebello-pontine angle epidermoid cyst operated on through a retrosigmoid approach A \& B: The preoperative DWI Diffusion and T2-weighted MRI showed a left cerebello-pontine angle epidermoid cyst extended to the petroclival region in a 30 years-old woman. The tumor was 
incidentally detected in imaging studies after a traumatic brain injury. The patient was free from any symptom before surgery. C: Peroperative view. The tumor was resected through the corridor between the acoustico-facial bundle and the lower CNs. The cranial nerves are exposed. D \& E: No CN disturbances occurred in the postoperative period. The DWI Diffusion and T2-weighted MR performed 3 months after surgery did not reveal any tumor residue, and remained stable 2 years after surgery. angle epidermoid cyst extended to the petroclival region in a 30 years-old woman. The tumor was incidentally detected in imaging studies after a traumatic brain injury. The patient was free from any symptom before surgery. C: Peroperative view. The tumor was resected through the corridor between the acoustico-facial bundle and the lower CNs. The cranial nerves are exposed. D \& E: No CN disturbances occurred in the postoperative period. The DWI Diffusion and T2-weighted MR performed 3 months after surgery did not reveal any tumor residue, and remained stable 2 years after surgery. 

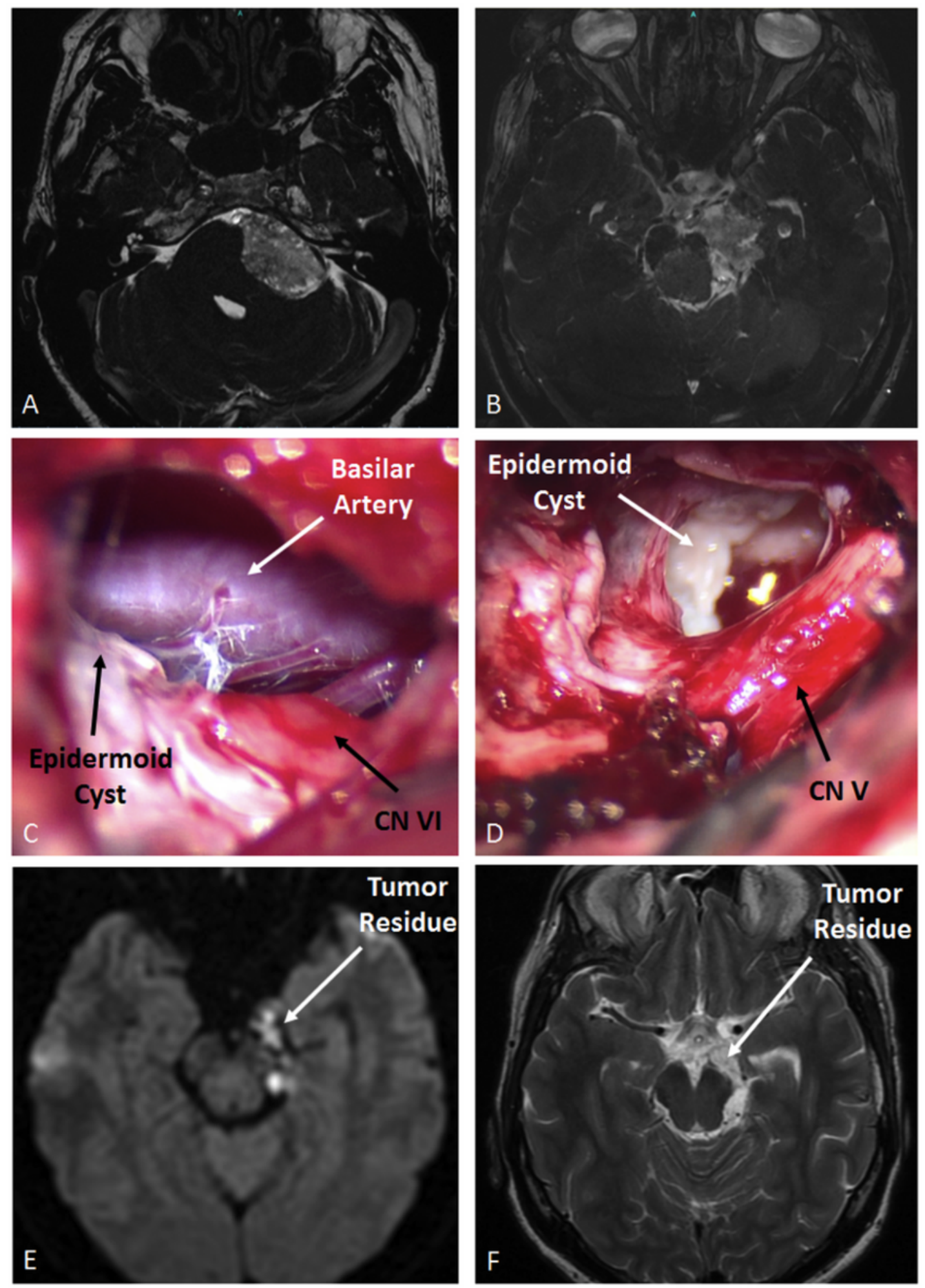

\section{Figure 2}

Illustrative case of a petroclival epidermoid cyst operated on through a transpetrosal approach. A \& B: The preoperative T2-weighted MRI showed a right petroclival epidermoid cyst (Figure 2A) in a 44 yearsold man. He presented with a slight numbness of $\mathrm{CN}$ V2 \& 3, and reported trigeminal neuralgia for 2 years. The superior pole of the tumor extended to the perimesencephalic cistern (Figure 2B). The tumor was responsible for a mass effect on the brainstem and the ipsilateral middle cerebellar peduncle. C \& D: 
Peroperative views. The operative window after epidural anterior transpetrosal approach offered an exposure from the trigeminal nerve to the basilar and the superior cerebellar artery. E \& F: The patient presented postoperative transient diplopia due to CN IV disturbances, and retained long term CN V3 facial hypoesthesia. The DWI Diffusion and T2-weighted MR performed 3 months after surgery showed small residual fragments of tumor located at the level of perimesencephalic cistern. Those remnants experienced asymptomatic regrowth 5 years after surgery. A Wait-and-reScan policy was decided.
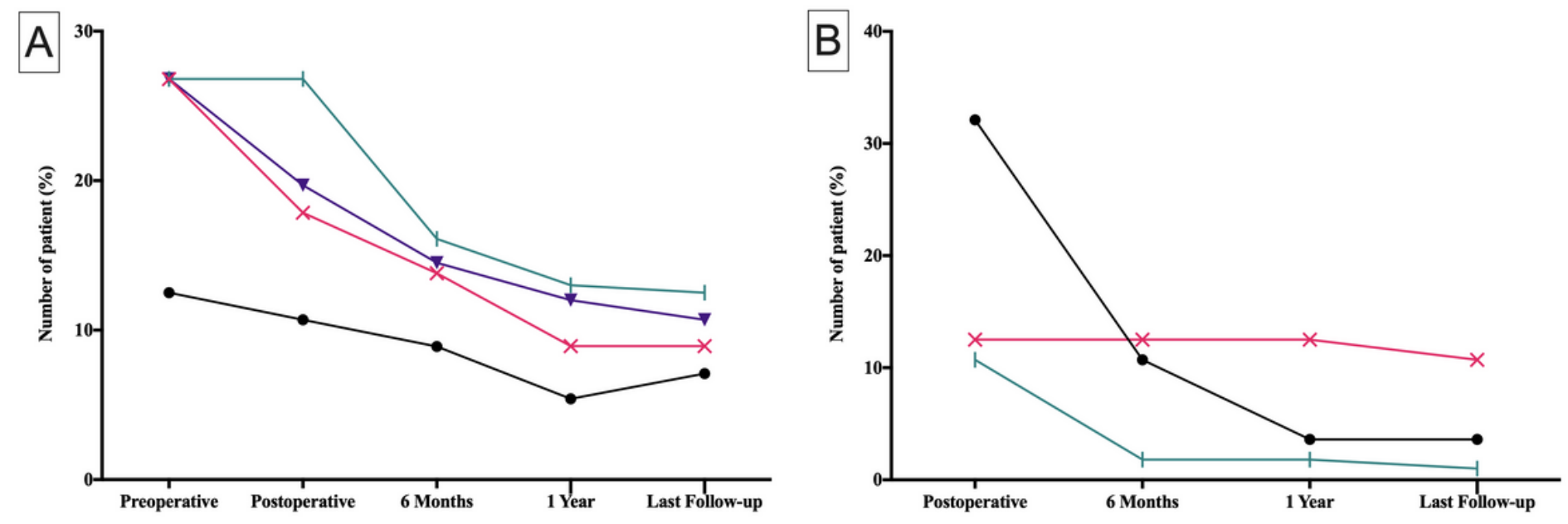

\section{Figure 3}

Evolution of cranial nerves deficit A: Evolution of cranial nerves preoperative deficit Line chart representing the proportion of patients with $\mathrm{CN}$ deficit during follow up among those with preoperative $\mathrm{CN}$ disorders. Only the most frequent $\mathrm{CN}$ deficits are illustrated: oculomotor (.), trigeminal hypoesthesia (+), trigeminal neuralgia $(x)$ and cochlear nerve $(v)$. B: Evolution of new postoperative cranial nerves deficit Line chart representing the proportion of patients with new $\mathrm{CN}$ deficit during follow up. Only the most frequent $\mathrm{CN}$ deficits are illustrated: oculomotor (.), cochlear (x) and lower cranial nerves (+). 


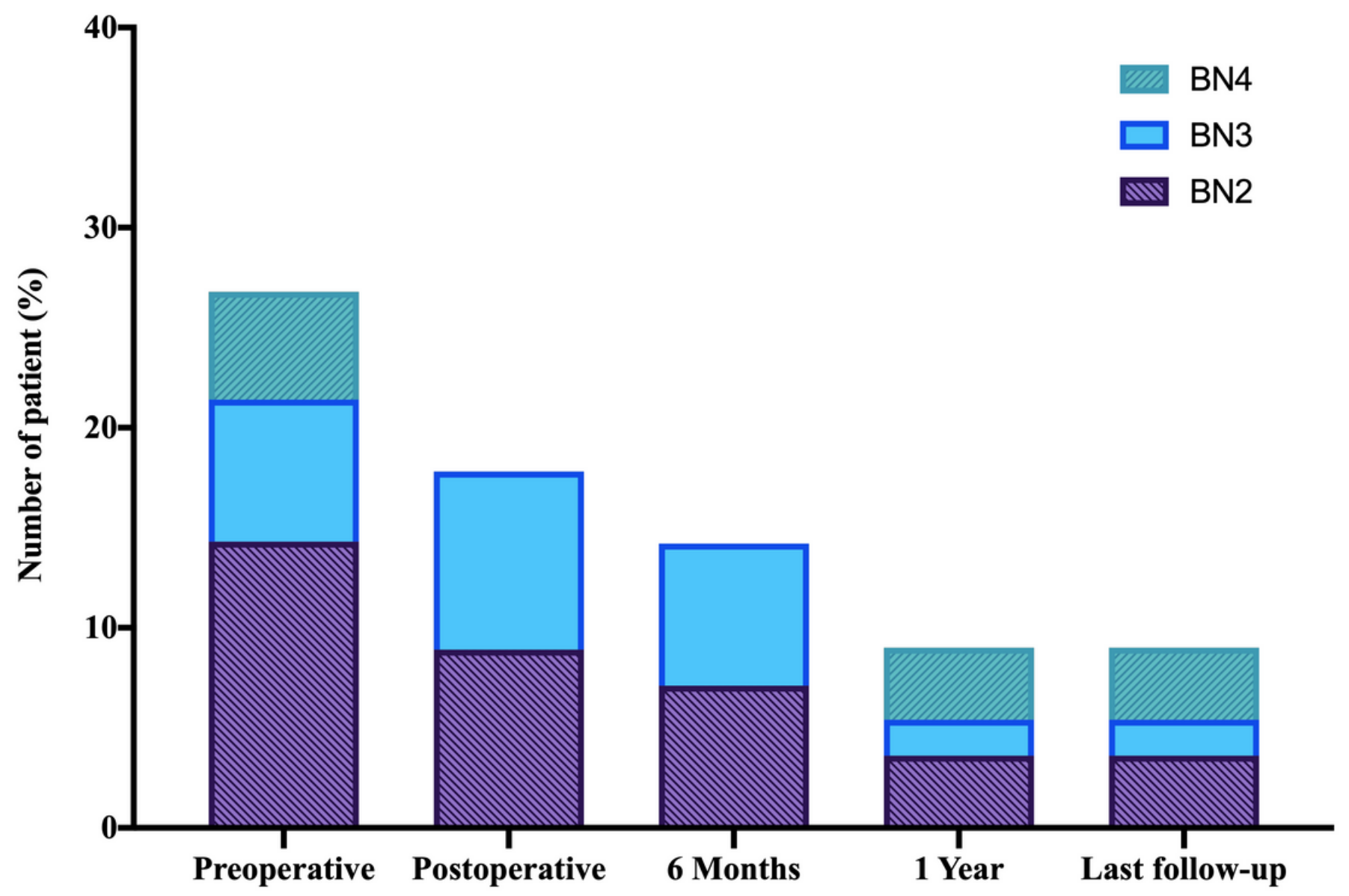

Figure 4

Evolution of preoperative trigeminal neuralgia Bar Chart illustrating the evolution of preoperative trigeminal neuralgia during follow-up, according to the Barrow Neurological Institute (BNI) Classification (from Grade 1 to Grade 5). Grade 1 defines asymptomatic patients, who are not shown is this figure. No Grade 5 patients was identified in our cohort. 

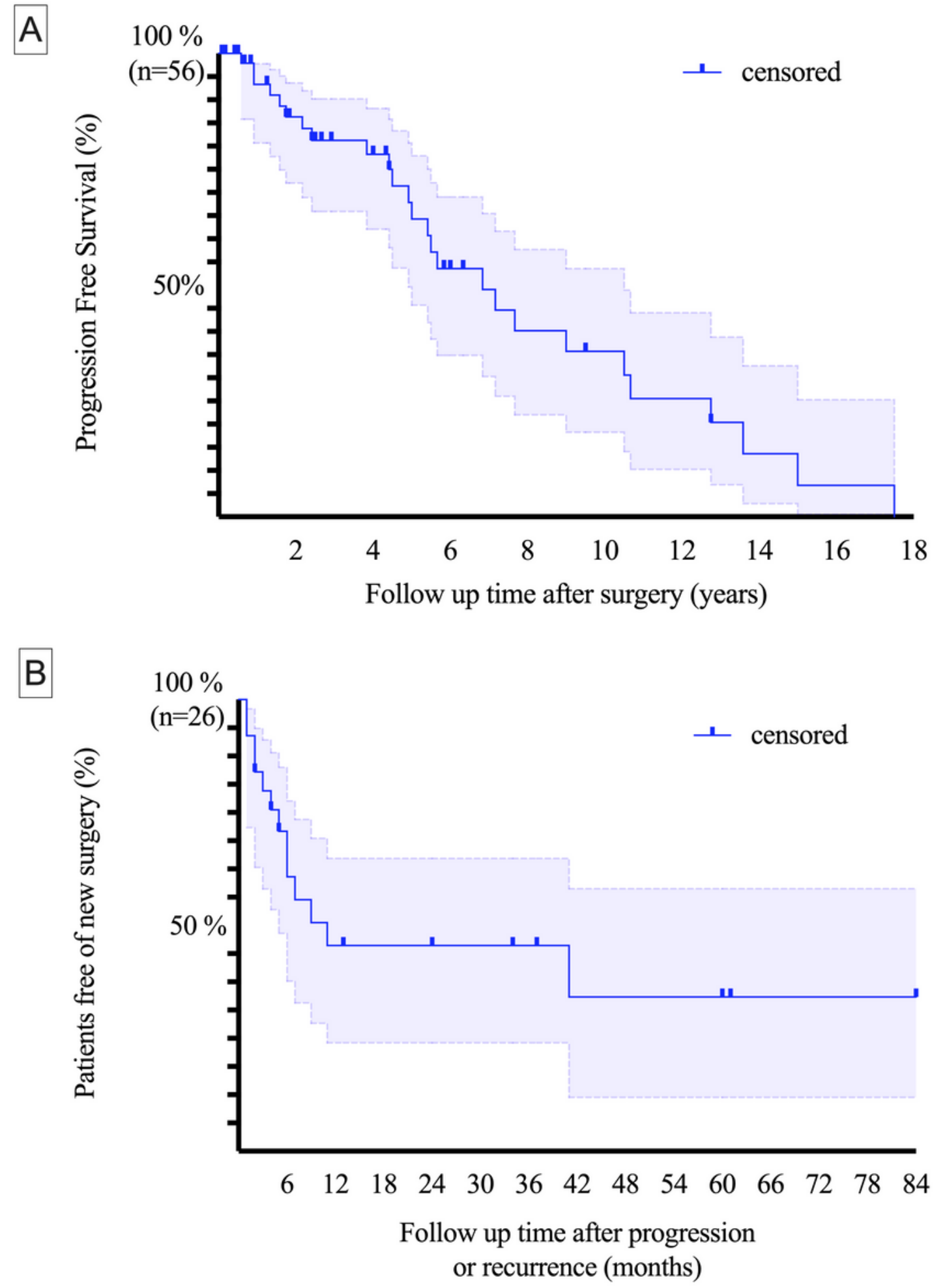

\section{Figure 5}

Tumor control A: Kaplan-Meier plot illustrating progression-free survival. The shaded areas indicate $95 \%$ $\mathrm{Cl}$. B: Kaplan-Meier plot illustrating the delay between progression/recurrence and a second resection surgery. The shaded areas indicate $95 \% \mathrm{Cl}$. 